\title{
Efficacy of Chuanxiong Ding Tong Herbal Formula Granule in the Treatment and Prophylactic of Migraine Patients: A Randomized, Double-Blind, Multicenter, Placebo-Controlled Trial
}

\author{
Caihong Fu, ${ }^{1}$ Lihua Yu, ${ }^{1}$ Yihuai Zou, ${ }^{1,2}$ Kegang Cao, ${ }^{1}$ Jianjun Zhao, ${ }^{3}$ Haiyang Gong, ${ }^{4}$ \\ Shuquan Zhang, ${ }^{5}$ Anji Lin, ${ }^{6}$ Mengjiu Dong, ${ }^{7}$ Wenming Yang, ${ }^{8}$ Tao Li, ${ }^{9}$ Liyun He, ${ }^{10}$ Fei Su, ${ }^{1}$ \\ Ruolan Wu, ${ }^{1}$ and Dongdong Lin ${ }^{1}$ \\ ${ }^{1}$ Department of Neurology, Dongzhimen Hospital, Beijing University of Chinese Medicine, No. 5 Haiyun Cang, Dong Cheng District, \\ Beijing 100700, China \\ ${ }^{2}$ Key Laboratory for Internal Chinese Medicine of Ministry of Education and Beijing, Beijing University of Traditional Chinese \\ Medicine, No. 5 Haiyun Cang, Dong Cheng District, Beijing 100700, China \\ ${ }^{3}$ Department of Neurology, The Affiliated Hospital to Changchun University of Chinese Medicine, No. 1478 Gongnong Avenue, \\ Chaoyang District, Changchun 130021, China \\ ${ }^{4}$ Department of Traditional Chinese Medicine, Beijing Tiantan Hospital, Capital Medical University, No. 6 Tiantan Xili, \\ Dong Cheng District, Beijing 100050, China \\ ${ }^{5}$ Department of Neurology, Taian Hospital of Traditional Chinese Medicine, No. 216 Yingxuan Avenue, Taian 271000, China \\ ${ }^{6}$ Department of Neurology, Xiamen Hospital of Traditional Chinese Medicine, No. 1739 Xianyue Road, Xiamen 361001, China \\ ${ }^{7}$ Department of Neurology, Hubei Hospital of Traditional Chinese Medicine, No. 4 Huayuan Shan, Wuchang District, Wuhan 430061, \\ China \\ ${ }^{8}$ Department of Neurology, The First Subsidiary Affiliated Hospital of Anhui College of Traditional Chinese Medicine, No.117 Meishan \\ Road, Hefei 230031, China \\ ${ }^{9}$ Department of Neurology, Xiyuan Hospital, China Academy of Chinese Medical Sciences, No. 1 Xiyuan Playground, Haidian District, \\ Beijing 100091, China \\ ${ }^{10}$ China Academy of Chinese Medical Sciences, No. 16 South Street, Dong zhimen nei, Dong Cheng District, Beijing 100700, China
}

Correspondence should be addressed to Yihuai Zou, zouyihuai2004@yahoo.com.cn and Kegang Cao, Kgdoctor@sina.com.cn

Received 25 July 2012; Accepted 24 September 2012

Academic Editor: Alan Bensoussan

Copyright ( $) 2012$ Caihong Fu et al. This is an open access article distributed under the Creative Commons Attribution License, which permits unrestricted use, distribution, and reproduction in any medium, provided the original work is properly cited.

Objective. To evaluate the efficacy of traditional Chinese herbal ChuanXiong Ding Tong herbal formula granule (CXDT-HFG) for migraine patients with "the Syndrome of Liver Wind and Blood Stasis." Methods. 150 migraine patients were recruited and assigned randomly in a double-blind, placebo-controlled study to receive CXDT-HFG $(n=99)$ plus necessary analgesics, or placebo $(n=$ 51) plus necessary analgesics for 16 weeks ( 12 weeks' intervention and 4 weeks' follow up). Outcome measures included migraine days, frequency of migraine attacks, analgesics consumption for acute treatment, and the proportion of responders as well as the visual analogue scale (VAS) scores and intensity for pain. Results. Compared with the placebo group, the CXDT-HFG group showed significant reduction in migraine days and attacks frequency at week 12 and follow-up period $(P<0.05)$ as well as in the reduction of VAS scores at follow-up period.There was significant difference in the proportion of responders between the two groups at follow-up period $(P=0.014)$. However there were no significant differences between the two groups in analgesics consumption $(P>0.05)$. Conclusion. CXDT-HFG was more effective than placebo in decreasing days of migraine attacks, frequency, VAS scores, and relieving pain intensity for migraine patients.

\section{Introduction}

Migraine, a common and chronic headache, which is understood to be a neurovascular dysfunction, is characterized with recurrent headache attacks. The pain is located unilaterally, moderate to severe intensity, usually aggravated by physical activity, and usually lasts $4-72$ hours. In addition, migraine attacks are often accompanied by nausea and/or vomiting, photophobia, phonophobia, etc. 
Migraine is high morbidity, procrastinating, and refractory, severely affecting the ability to work and quality of life [15]. The epidemiological study from the United States shows that the lifelong cumulative inDcidence of migraine is $7.4 \%$ in males and $21 \%$ in females [6]. The temporary functional disability, accompanying migraine [6], brings huge economic losses to the society. Migraine has been listed as one of the most serious, chronic, and dysfunctional diseases, which is equal to quadriplegia, mental disorders, and dementia by World Health Organization [7]. Due to its high incidence and high economic cost [2], the treatment and prevention of migraine has already attracted broad attention in the world in recent years.

Migraine is divided into the period of acute episode and chronic remission [8] and its treatment and prevention requires standard drug therapy and regular management [912]. There are many drugs used to treat acute migraine, such as aspirin, acetaminophen, nonsteroidal, anti-inflammatory drugs (NSAIDs), dihydroergotamine, and the triptans. Meanwhile Beta-blockers, calcium channel blockers, tricyclic antidepressant, and antiepileptic drugs have been used for migraine prevention $[4,13,14]$. However, in clinical practice it has been shown that these drugs can lead to headache or increase the frequency of migraine attacks and other side effects $[15,16]$, and which, to some extent, limit their application to migraine sufferers.

In the United States, Chinese herbal medicine, acupuncture and other natural therapy, have been licensed for use, and it is recognised clinical practice for herbal medicine to hold a place of importance in remedying headaches effectively and safely [17]. Clinical studies show that traditional acupuncture therapy can effectively reduce the migraine days and acute pain drug consumption, especially for migraine prevention, but no statistical differences are seen in the improvement of pain intensity $[18,19]$, thus sufferers still need drug treatment in the treatment and prevention of migraine. However, those studies on using Chinese herbal medicine to treat migraine with the method of multicenter, prospective, randomized, placebo controlled, double blind, are relatively few. According to the Guidelines for controlled trials of drugs in migraine (second edition) [20], this study chose migraine patients with "the Syndrome of Liver Wind and Blood Stasis" commonly seen in traditional Chinese medicine (TCM) clinic, and Chuanxiong Ding Tong herbal formula granule (CXDT-HFG) as treatment drug, so as to further evaluate the efficacy of Chinese herbal medicine in the treatment and prevention of migraine.

\section{Materials and Methods}

2.1. Study Design. A multicenter, prospective, centralrandomized, double-blind, placebo-controlled trial was conducted in this study. Migraine patients who met the inclusion criteria were randomly assigned into the experimental group and control group in a 2:1 ratio. The experimental group was treated with Chinese herbal medicine and the placebo group with placebo. Both groups were permitted to use the necessary analgesics during migraine acute attacks, as well as accepted regular management. The retrospective historical record of migraine attacks for nearly 3 months was defined as the baseline of the study. The study spans 16 weeks, including a treatment period of 12 weeks, and a follow-up period of 4 weeks. Headache diary was given to patients to record the details (migraine days, frequency, visual analogue scale (VAS) scores and acute medication, etc.) of migraine attacks during the trial period. These outcome measures such as migraine days, frequency of migraine attacks, VAS scores and intensity for pain were evaluated at 4 weeks, 8 weeks, 12 weeks, and follow-up period Figure 1.

This study was designed and carried out cooperatively by methodologists and statisticians of the China Academy of Chinese Medical Sciences in Beijing. And the study protocol conforms to the Helsinki Declaration [21] and the research regulations for Chinese clinical trials. The Ethics Committee of the Affiliated Dongzhimen Hospital of Beijing University of Chinese Medicine reviewed and approved the study protocol. All participants signed informed consent before enrolment.

2.2. Setting and Participants. 150 migraine patients with "the Syndrome of Liver Wind and Blood Stasis" were recruited between January 2008 and June 2011 from outpatient departments in the following 8 hospitals: Dongzhimen Hospital affiliated to the Beijing University of Traditional Chinese Medicine, the Affiliated Hospital to Changchun University of Chinese Medicine, Beijing Tiantan Hospita affiliated to the Capital Medical University, Tai an Hospital of Traditional Chinese Medicine, Xiamen Hospital of Traditional Chinese Medicine, Hubei Hospital of Traditional Chinese Medicine, The First Subsidiary Hospital of Anhui College of Traditional Chinese Medicine, Xiyuan Hospital affiliated to the China Academy of Chinese Medical Sciences. All patients, with diagnosis of migraine with aura or without aura in accordance with the 2nd edition of the International Headache Society's International Classification of Headache Disorders (ICHD-II, IHS 2004) [8]. The study had a previous 3 months of retrospective record of migraine attacks (referred to as baseline) and 12-week double-blind, parallel group, placebocontrolled phase with trial drug treatment at day " 0 " of the double-blind phase, follow-up visit for 4 weeks after the experimental drug withdrawn (details will be described separately).

2.3. Diagnostic Criteria. The diagnostic criteria for this study were as follows. (1) Diagnosis standard in Western medicine: migraine without aura $(\mathrm{MO})$ or with a typical aura (MA) as defined by 2004 HIS [8]. (2) TCM Differentiation standard: in accordance with the guiding principles for the clinical study of new drugs for use in traditional Chinese medicine released in 2002, combined with the characteristics of migraine, the standard of "Liver Wind and Blood Stasis syndrome" as follows: headache in the left or right, repeated attacks, severe pain, lasting for several hours or days, accompanyed by nausea, vomiting, vertigo, etc.; dark red or dark purple tongue, or tongue has bruises, ecchymosis or stasis points, thin-white fur, wiry pulse. 


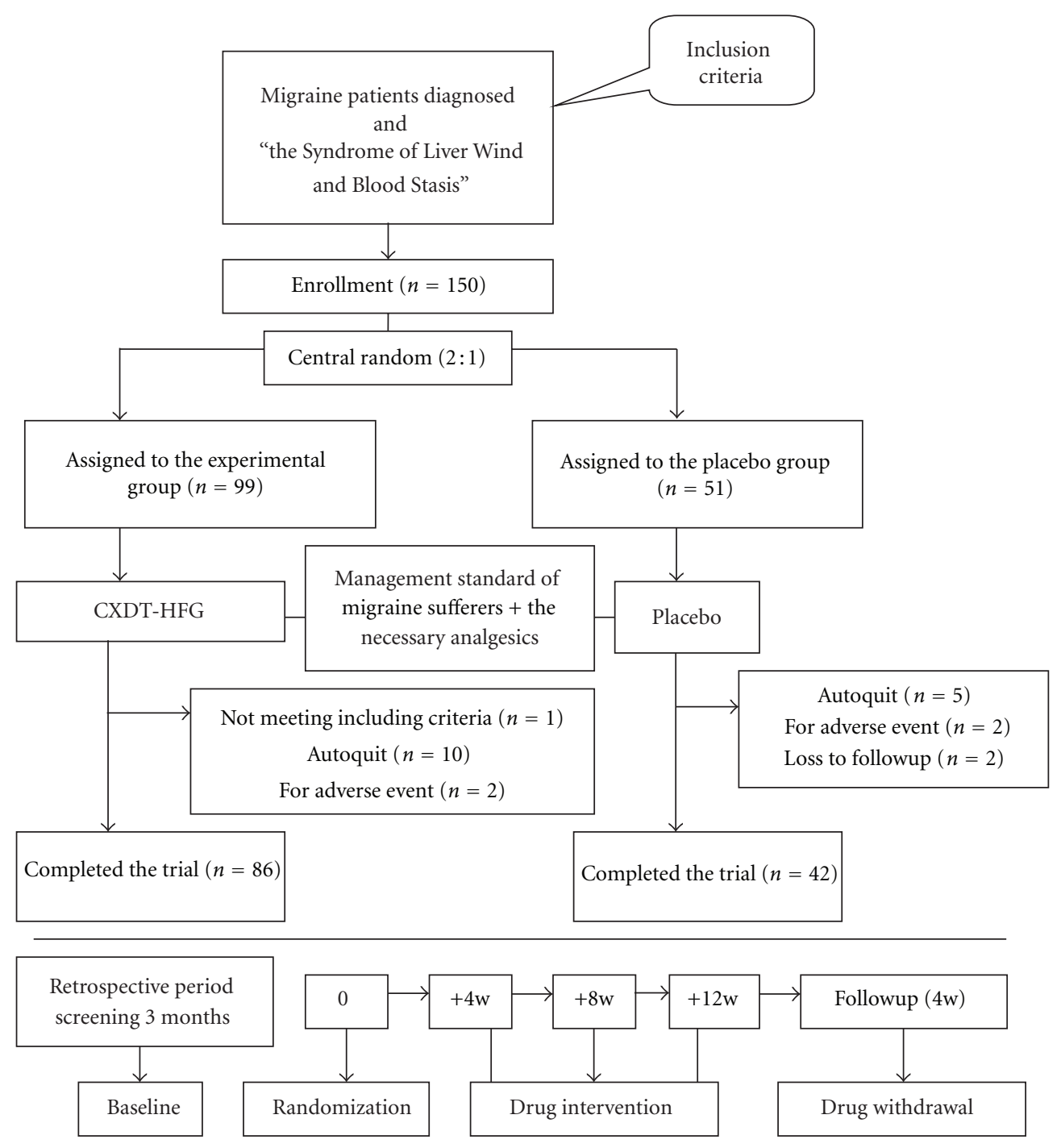

FIGURE 1: Flow diagram of the study progress about enrollment, randomization, intervention, and completion of the trial.

2.4. Inclusion Criteria. The inclusion criteria for this study were as follows: (1) diagnosed as migraine without aura (MO) or with a typical aura (MA) according to the diagnostic criteria specified by the International Classification of Headache Disorders; (2) meet the diagnostic standard of "Liver Wind and Blood Stasis syndrome" in TCM; (3) age of first onset $\leq 50$ years old; (4) the history of migraine $>1$ year; (5) with more than 6 times of migraine attacks in the previous 3 months; (6) age between 18 and 65 years old; (7) the patients voluntarily joined this study with informed consent.

2.5. Exclusion Criteria. The exclusion criteria were as follows: (1) times of using analgesics for acute headache $>10$ times per month; (2) alcohol or other drug abuse; (3) primary disease of the liver, kidney, hematopoietic system, cardiovascular system, or cerebrovascular system; (4) psychiatric conditions; (5) hypersensitivity to the trial drug; (6) pregnancy and lactation.
2.6. Interventions. The patients in the experimental group would be provided with CXDT-HFG, while the control group would take placebo. Both of them were provided by Hua Run San-Jiu Pharmaceutical Co. LTD. Mix the drugs or placebo with warm-water, and take them twice daily (separately taken in the morning and evening). Both groups were permitted to use the necessary analgesics during an acute migraine attack as well as accepted regular management.

The experimental drug CXDT-HFG is composed of Chuanxiong Rhizoma (Chuanxiong, $12 \mathrm{~g}$ ), Cyathulae Radix (Chuan Niuxi, $10 \mathrm{~g}$ ), Dioscoreae Hypoqlaucae Rhizoma (Chuan Bixie, 20 g), Chrysanthemi Flos (Juhua, 6 g), Uncaria rhynchophylla Pamuluscum Uncis (Gouteng, $20 \mathrm{~g}$ ), Tribuli terrestris Fructus (Bai Jili, $10 \mathrm{~g}$ ), Coicis Semen (Yi Yiren, $20 \mathrm{~g}$ ), Amomi FructusRotundus (Bai Doukou, 6 g), Pinelliae Rhizoma Preparatum (Zhi Banxia, 6 g).

The placebo of single Chinese medicine formula is consisted of dextrin, lactose, caramel pigment, and bitters. 
Caramel pigment is provided by Shanghai Love Food Industry Company and bitters are manufactured by Zhejiang Deere pharmaceutical factory.

There are better similarities in some ways of dosage, shape, and predominant flavor between the experimental drug CXDT-HFG and placebo.

Permitted medication and specification: generally the patients who have 2 days of headache attacks per week will be permitted to use acute drug treatment; patients should choose the analgesics commonly used without the effect of migraine prevention; the intensity of headache should be measured by VAS and patients should choose corresponding analgesics according to different pain degree. All analgesics used and their doses and effect should be recorded in the diary.

Management standard of participants: (1) patients need to record the headache diary seriously so as to fully understand the time and characteristics of headache attacks. (2) Patients should have a regular lifestyle, the work and rest on schedule, quit smoking, and moderate alcohol consumption. (3) Regulate and refresh emotion and avoid disposition stimulation. (4) Light diet, avoid spicy, and greasy food, reduce the intake of chocolate, coffee, cheese, and other contour tyrosine foods and sausage, ham, hot dogs, and other bacon meats. (5) Patients need to do timely medical examination and treatment if the nature, the degree, and the frequency of headache have changed.

2.7. Outcome Measures. The outcome measures included the changes of migraine days, attack frequency, analgesic consumption, and the proportion of responders (defined as the proportion of patients with a reduction of migraine days and times by at least $50 \%[18,20]$ ) from baseline to weeks $4,8,12$ and follow-up period. The frequency of migraine attacks is the main evaluation index, which indicates improvement of the migraine to the maximum extent [22]. Thus the frequency of migraine attacks per 4 weeks should be the primary efficacy measure. The secondary outcome measure was the proportion of responders in attack frequency and migraine days during treatment and followup period compared with the baseline period. Inaddition, VAS scores and intensity for pain (mild, moderate, and severe) were assessed at weeks 4, 8, 12 and follow-up period (because VAS scores and pain intensity were not recorded in headache diary retrospectively for nearly 3 months, the 4 weeks' VAS scores and intensity would be seen as baseline at this point). Additionally, patients were required to record adverse events in their headache diaries.

Patients filled in a headache diary on time and recorded the time, frequency, location (the forehead, top, temporal, and back of the head), VAS scores, intensity (mild, moderate, and severe), types of pain, analgesics consumption during migraine acute attacks, and concomitant symptom (nausea, vomiting, photophobia, phonophobia, etc.) in detail, as follows: (1) the days of migraine attacks refer to the onset of the migraine days (provided migraine attack in one day, it will be deemed to one day); (2) a migraine attack that was interrupted either by sleep or treatment but relapsed within
48 hours was required to be documented as a single attack. (3) VAS scores (quantitative index for pain): let the patient himself point out that most representative number of pain; (4) Pain Intensity on the basis of the Numeric Rating Scale (NRS-11) which has been widely used for the assessment of pain [23], was graded according to the number represent pain that patients point out from the scaleplate of VAS: 0 stands for no headache, score range $0 \sim 4$ stands for mild headache, score range $4 \sim 7$ stands for moderate headache, score range $7 \sim 10$ stands for severe headache.

2.8. Statistical Methods. Statistical analysis was performed with SPSS (13.0) program for Windows (SPSS, Chicago, IL, USA). Data was managed through the online facility established by the Traditional Chinese Medicine Clinical Foundation Institute of medicine of the China Academy of the Chinese Medical Sciences, and was analyzed on the full analysis set (FAS), and the per-protocol set (PPS) for adherence. The result of data analysis was mainly for PPS in this trial. The measurement data were expressed as mean \pm standard deviation, to check the data of all groups with normal test and homogeneity of variance test. Data analysis was performed by nonparametric statistics to these unmatched normally distributed measurements, including age, course of disease, migraine days, and attacks frequency, etc., which were compared between groups using Mann-Whitney $U$ test. Results were shown with 95\% confidence interval (95\% CI). Measurement data before and after treatment were compared using ANOVA for repeated measures. Headache intensity classification and the proportion of responders were analyzed by $\chi^{2}$ test. The level of significance was set at 0.05 , if $P<0.05$, there were statistical differences.

\section{Results}

3.1. Dropouts. During the study, 22 patients dropped out, a rate of $14.7 \%$ (13 from the CXDT-HFG group $13.1 \%$ and 9 from the placebo group 17.6\%). Among these, 1 patient was rejected for not meeting inclusion criteria (1 from the CXDT-HFG group), 15 patients dropped out during the treatment period (10 from the CXDT-HFG group, 5 from the placebo group) because of lack of efficacy, pregnancy, etc. And 4 patients dropped out for adverse events ( 2 from the CXDT-HFG group, 2 from the placebo group). The other 2 patients were lost to followup due to change of contact information ( 2 from the placebo group). The reasons for the dropouts in the 2 groups are detailed in Figure 1.

3.2. Characteristics of Demography and Baseline. The demographic and baseline parameters with the PPS population were shown in Table 1, which showed that the 2 groups were comparable at baseline. The Table 1 summarizes the main baseline characteristics of the 128 patients based on PPS. Participants of CXDT-HFG group had a mean age of 35.77 years and $73.3 \%$ (63) were women while the placebo group had a mean age of 34.58 years and $73.8 \%$ (31) were women. There were no statistical differences $(P>0.05)$ between the two groups in migraine days, attack frequency, 
TABLE 1: Baseline characteristics of the study participants and primary outcome measures (PPS, $N=128$ ).

\begin{tabular}{|c|c|c|c|c|c|}
\hline & \multicolumn{2}{|c|}{ CXDT-HFG group $(n=86)$} & \multicolumn{2}{|c|}{ Placebo group $(n=42)$} & \multirow{2}{*}{$P^{*}$ values } \\
\hline & Mean \pm SD & $95 \% \mathrm{CI}$ & Mean \pm SD & $95 \% \mathrm{CI}$ & \\
\hline Age, years & $35.77 \pm 11.60$ & $(33.28,38.25)$ & $34.58 \pm 9.85$ & $(31.52,37.65)$ & 0.738 \\
\hline \multicolumn{6}{|l|}{ Sex } \\
\hline Male, $n(\%)^{\#}$ & $23(26.7 \%)$ & - & $11(26.2 \%)$ & - & 0.947 \\
\hline Female, $n(\%)$ & $63(73.3 \%)$ & - & $31(73.8 \%)$ & - & - \\
\hline Course of disease (months) & $86.26 \pm 88.10$ & $(67.37,105.14)$ & $82.12 \pm 72.76$ & $(59.45,104.79)$ & 0.716 \\
\hline \multicolumn{6}{|l|}{ Migraine days ${ }^{\S}$} \\
\hline Baseline & $4.48 \pm 4.12$ & $(3.59,5.36)$ & $4.13 \pm 4.33$ & $(2.78,5.47)$ & 0.572 \\
\hline $4 \mathrm{w}$ & $4.01 \pm 5.11$ & $(2.92,5.11)$ & $2.88 \pm 2.05$ & $(2.24,3.52)$ & 0.548 \\
\hline Improvement from baseline & $-0.46 \pm 4.36$ & $(-1.40,0.47)$ & $-1.25 \pm 4.89$ & $(-2.77,0.28)$ & 0.755 \\
\hline $8 \mathrm{w}$ & $2.41 \pm 3.34$ & $(1.69,3.12)$ & $1.93 \pm 1.52$ & $(1.45,2.40)$ & 0.848 \\
\hline Improvement from baseline & $-2.07 \pm 4.42$ & $(-3.01,-1.12)$ & $-2.20 \pm 4.77$ & $(-3.68,-0.71)$ & 0.438 \\
\hline $12 \mathrm{w}$ & $1.44 \pm 3.22$ & $(0.75,3.22)$ & $1.93 \pm 2.28$ & $(1.22,2.64)$ & 0.054 \\
\hline Improvement from baseline & $-3.04 \pm 5.12$ & $(-4.13,-1.93)$ & $-2.20 \pm 4.95$ & $(-3.74,-0.66)$ & $0.033^{\triangle}$ \\
\hline Follow-up period & $1.06 \pm 3.13$ & $(0.39,1.73)$ & $1.69 \pm 2.35$ & $(0.96,2.42)$ & $0.019^{\ddagger}$ \\
\hline Improvement from baseline & $-3.42 \pm 5.04$ & $(-4.49,-2.34)$ & $-2.44 \pm 4.94$ & $(-3.97,-0.90)$ & $0.042 \triangle$ \\
\hline \multicolumn{6}{|l|}{ Migraine attack frequency ${ }^{\S}$} \\
\hline Baseline & $3.82 \pm 2.16$ & $(3.36,4.28)$ & $3.91 \pm 4.32$ & $(2.57,5.26)$ & 0.350 \\
\hline $4 \mathrm{w}$ & $2.55 \pm 2.00$ & $(2.12,2.97)$ & $2.12 \pm 1.21$ & $(1.74,2.50)$ & 0.430 \\
\hline Improvement from baseline & $-1.27 \pm 1.63$ & $(-1.62,-0.92)$ & $-1.79 \pm 4.52$ & $(-3.20,-0.39)$ & 0.641 \\
\hline $8 \mathrm{w}$ & $1.74 \pm 1.57$ & $(1.41,2.08)$ & $1.52 \pm 1.13$ & $(1.17,1.88)$ & 0.686 \\
\hline Improvement from baseline & $-2.07 \pm 2.03$ & $(-2.51,-1.64)$ & $-2.39 \pm 4.65$ & $(-3.84,-0.94)$ & 0.404 \\
\hline $12 \mathrm{w}$ & $0.98 \pm 1.35$ & $(0.69,1.27)$ & $1.24 \pm 1.08$ & $(0.90,1.57)$ & 0.085 \\
\hline Improvement from baseline & $-2.84 \pm 2.36$ & $(-3.35,-2.33)$ & $-2.67 \pm 4.60$ & $(-4.11,-1.24)$ & $0.043 \triangle$ \\
\hline Follow-up period & $0.74 \pm 1.32$ & $(0.46,1.03)$ & $1.19 \pm 1.35$ & $(0.77,1.61)$ & $0.033^{\ddagger}$ \\
\hline Improvement from baseline & $-3.08 \pm 2.32$ & $(-3.57,-2.58)$ & $-2.72 \pm 4.63$ & $(-4.17,-1.28)$ & $0.033 \triangle$ \\
\hline \multicolumn{6}{|c|}{ Acute pain drug consumption (times) $)^{\S}$} \\
\hline Baseline & $0.62 \pm 1.33$ & $(0.33,0.91)$ & $0.49 \pm 1.10$ & $(0.15,0.83)$ & 0.506 \\
\hline $4 \mathrm{w}$ & $0.35 \pm 1.19$ & $(0.09,0.60)$ & $0.26 \pm 0.83$ & $(0.00,0.52)$ & 0.888 \\
\hline $8 \mathrm{w}$ & $0.17 \pm 0.93$ & $(-0.02,0.38)$ & $0.12 \pm 0.45$ & $(-0.02,0.26)$ & 0.627 \\
\hline $12 \mathrm{w}$ & $0.08 \pm 0.47$ & $(-0.02,0.19)$ & $0.14 \pm 0.57$ & $(-0.03,0.32)$ & 0.371 \\
\hline Follow-up period & $0.02 \pm 0.15$ & $(-0.01,0.06)$ & $0.21 \pm 0.75$ & $(-0.02,0.45)$ & 0.066 \\
\hline \multicolumn{6}{|l|}{ Responder rate (migraine days)\# } \\
\hline $12 \mathrm{w}$ & $62(72.1 \%)$ & - & $28(66.7 \%)$ & - & 0.528 \\
\hline Follow-up period & $72(83.7 \%)$ & - & $27(64.3 \%)$ & - & $0.014^{\ddagger}$ \\
\hline \multicolumn{6}{|l|}{ Responder rate (attack frequency) } \\
\hline $12 \mathrm{w}$ & $70(81.4 \%)$ & - & $29(69.0 \%)$ & - & 0.117 \\
\hline Follow-up period & $75(87.2 \%)$ & - & $31(73.8 \%)$ & - & 0.059 \\
\hline
\end{tabular}

w: weeks.

CI: confidence interval; PPS: per-protocol sets; significant difference, $P<0.05$. Data presented as mean \pm SD, number (percentage) and $95 \%$ CI.

* $P$ for comparison with control group.

${ }^{*} P$ values based on Chi-square test.

$\S_{P}$ values based on repeated measures.

${ }^{\ddagger} P<0.05$, for date comparison between groups.

$\triangle P<0.05$, for $D$ value from baseline comparison between groups.

course of disease, consumption of analgesics, all the baseline characteristics of the 2 groups were similar (Table 1 ).

3.3. Days of Migraine Attacks. The baseline of migraine days in the CXDT-HFG group was $4.48 \pm 4.12$ days and $4.13 \pm$
4.33 days in the placebo group. At 12 weeks and followup period, the migraine days in the CXDT-HFG group decreased to 1.44 days and 1.06 days, respectively, whereas in the placebo group, the migraine days reduced to 1.93 days and 1.69 days, respectively. In other words, the mean reduction of migraine days in the CXDT-HFG group were 
TABLE 2: Changes in VAS scores and pain intensity. (PPS, $N=128$ ).

\begin{tabular}{|c|c|c|c|c|c|}
\hline Variable & Group & $4 \mathrm{w}$ & $8 \mathrm{w}$ & $12 \mathrm{w}$ & Follow-up period \\
\hline \multirow{3}{*}{ VAS scores $\$$} & CXDT-HFG group $(n=86)$ & $4.96 \pm 2.01$ & $4.02 \pm 2.33$ & $2.39 \pm 2.41$ & $1.66 \pm 2.15$ \\
\hline & Placebo group $(n=42)$ & $4.90 \pm 2.09$ & $4.12 \pm 2.41$ & $3.21 \pm 2.45$ & $2.92 \pm 2.51$ \\
\hline & $P^{*}$ values & 0.893 & 0.679 & 0.060 & $0.005^{\ddagger}$ \\
\hline \multirow{11}{*}{ Pain intensity } & CXDT-HFG group $(n=86)$ & & & & \\
\hline & No pain, $n(\%)$ & $7(8.14 \%)$ & $15(17.44 \%)$ & $37(43.02 \%)$ & $49(56.98 \%)$ \\
\hline & Mild, $n(\%)$ & $15(17.44 \%)$ & $24(27.91 \%)$ & $27(31.40 \%)$ & $23(26.74 \%)$ \\
\hline & Moderate, $n(\%)$ & $57(66.28 \%)$ & $43(50.00 \%)$ & $21(24.42 \%)$ & $13(15.12 \%)$ \\
\hline & Severe, $n(\%)$ & $7(8.14 \%)$ & $4(4.65 \%)$ & $1(1.16 \%)$ & $1(1.16 \%)$ \\
\hline & Placebo group $(n=42)$ & & & & \\
\hline & No pain, $n(\%)$ & $4(9.52 \%)$ & $8(19.05 \%)$ & $12(28.57 \%)$ & $16(38.10 \%)$ \\
\hline & Mild, $n(\%)$ & $9(21.43 \%)$ & $13(30.95 \%)$ & $11(26.19 \%)$ & $10(23.81 \%)$ \\
\hline & Moderate, $n(\%)$ & $25(59.52 \%)$ & $19(45.24 \%)$ & $19(45.24 \%)$ & $16(38.10 \%)$ \\
\hline & Severe, $n(\%)$ & $4(9.52 \%)$ & $2(4.76 \%)$ & $0(0.00 \%)$ & $0(0.00 \%)$ \\
\hline & $P^{*}$ values & 0.684 & 0.673 & $0.037^{\ddagger}$ & $0.013^{\ddagger}$ \\
\hline
\end{tabular}

W: weeks. Significant difference, $P<0.05$. Data presented as mean $\pm \mathrm{SD}$, number (percentage).

${ }^{*} P$ for comparison with control group.

${ }^{\#} P$ values based on Chi-square test.

$\$ P$ values based on repeated measures.

${ }^{\ddagger} P<0.05$. Statistical differences for comparison between groups.

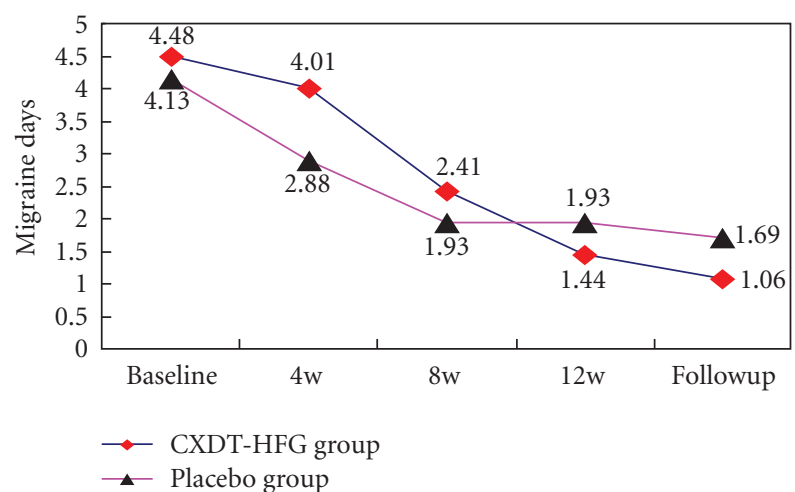

FIGURE 2: The trend of the changes for migraine days (PPS).

3.04 days, compared with 2.20 days in the placebo group at 12 weeks ( $95 \% \mathrm{CI},-4.13$ to -1.93 versus $95 \% \mathrm{CI},-3.74$ to $-0.66 ; P=0.033$ ), and a mean reduction of 3.42 days in the CXDT-HFG group compared with 2.44 days in the placebo group at follow-up period ( $95 \% \mathrm{CI},-4.49$ to -2.34 versus 95\% CI, -3.97 to $-0.90 ; P=0.042$ ).

And there was significant difference in the proportion of patients with a reduction of migraine days by at least $50 \%$ between the CXDT-HFG group and the placebo group at follow-up period $(83.7 \%$ versus $64.3 \%, P=0.014$ ) (Table 1; Figure 2).

3.4. Frequency of Migraine Attacks. The baseline of migraine attack frequency in the CXDT-HFG group is $3.82 \pm 2.16$ times and $3.91 \pm 4.32$ times in the placebo group. After 12 weeks' treatment, the frequency of migraine attacks of the CXDTHFG group reduced 2.84 times, while the placebo group reduced 2.67 times ( $95 \% \mathrm{CI},-3.35$ to -2.33 versus $95 \% \mathrm{CI}$,

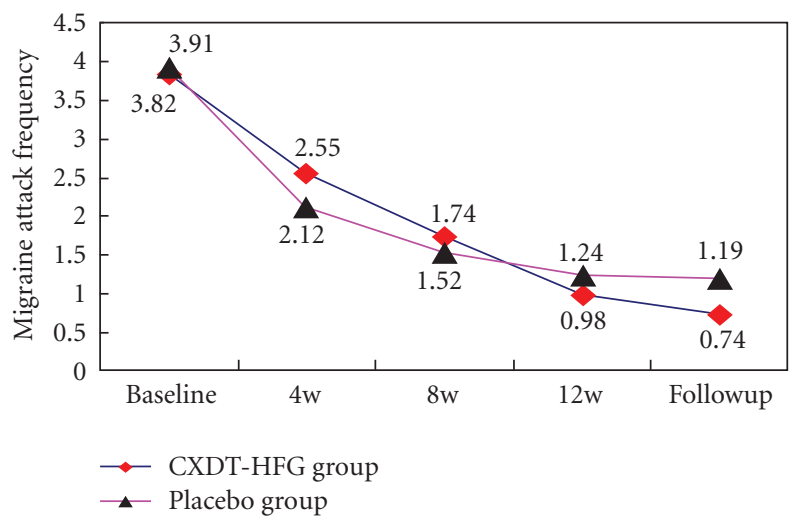

FIGURE 3: The trend of the changes for migraine attack frequency (PPS).

-4.11 to $-1.24 ; P=0.043)$. At follow-up period the attack frequency of the CXDT-HFG group reduced 3.08 times from baseline and the placebo group reduced 2.72 times (95\% $\mathrm{CI},-3.57$ to -2.58 versus $95 \% \mathrm{CI},-4.17$ to -1.28 ; $P=$ $0.033)$. However, no significant difference was found in the proportion of patients with a reduction of migraine attack frequency by at least $50 \%$ between the CXDT-HFG group and placebo group (Table 1; Figure 3).

3.5. VAS Scores and Pain Intensity. The mean VAS scores have significantly decreased in the CXDT-HFG group from $4.96 \pm 2.01$ at 4 weeks to $1.66 \pm 2.15$ at follow-up period, whereas in the placebo group from $4.90 \pm 2.09$ at 4 weeks to $2.92 \pm 2.51$ at follow-up period $(P=0.005)$. According to VAS scores, the pain degree is divided into four levels with no pain, mild, moderate, and severe. At 12 weeks and 


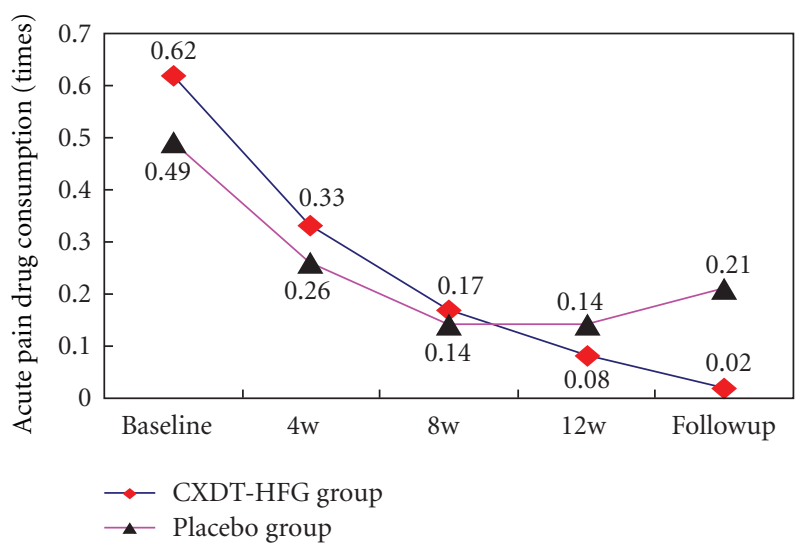

FIgure 4: The trend in the change for acute pain drug consumption (PPS).

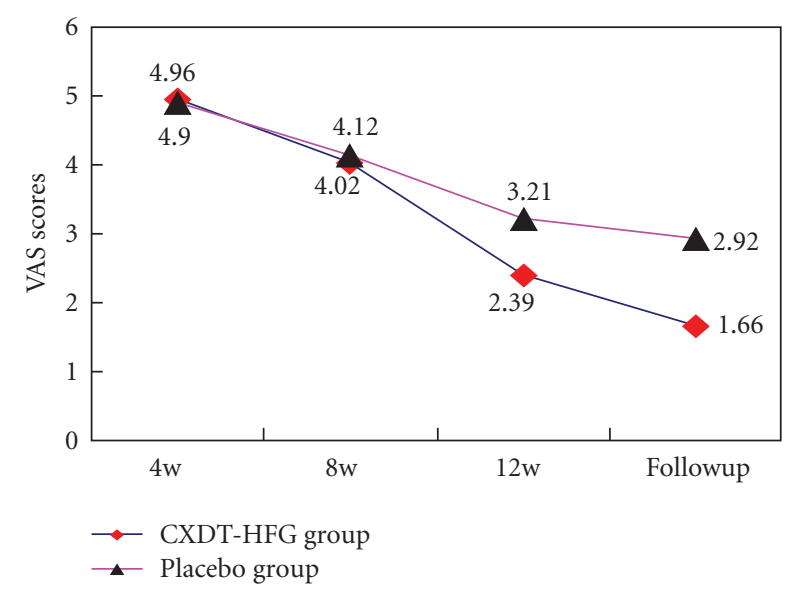

FigURE 5: Mean changes of VAS scores between 2 groups (PPS).

follow-up period, there were significant differences between the 2 groups about the pain degree $(P=0.037, P=0.013)$ (Table 2; Figures 5 and 6).

3.6. Acute Medication. The number of patients using acute pain drugs, such as aspirin, phenacetin, or ibuprofen, etc., has no significant difference between the 2 groups from baseline to follow-up period $(P>0.05)$ (Table 1; Figure 4).

3.7. Safety and Tolerability. A total of $15(11.72 \%)$ subjects in the randomized population experienced mild adverse events during the study period. Treatment-related adverse events were reported in $11.63 \%$ of the CXDT-HFG group (10 patients) and in $11.90 \%$ of the placebo group (5 patients). The main adverse events were as follows, maculopapula, eyelid edema, palpitations, abdominal pain and nausea, and they were resolved without sequelae after treatment was withdrawn. No clinically significant serious adverse events were reported in subjects on the aspects of liver and renal functions, blood, and urine routines before and after treatment.

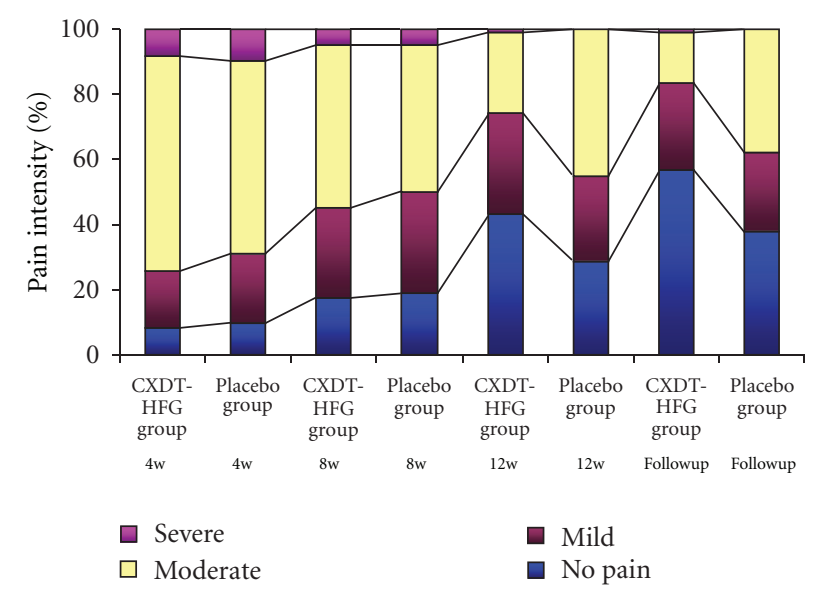

FIGURE 6: The percentage of pain intensity during the study (PPS).

\section{Discussion}

4.1. Summary of Main Findings. This trial revealed Traditional Chinese herbal CXDT-HFG to be better than placebo in reducing the days of migraine attacks, frequency, and pain intensity at 12 weeks and follow-up period $(P<0.05)$ as well as in the reduction of VAS scores at follow-up period. And along with the extension of treatment time, the days and frequency of migraine attacks and pain intensity still improved in the CXDT-HFG group. Whereas no significant difference was found between the 2 groups in consumption of acute pain drug (Figures 2, 3, 4, 5, 6).

At the follow-up period, we found that the placebo group presented rebound trend in migraine days and frequency, meanwhile the number of analgesics increased gradually. However the efficacy of the CXDT-HFG group continued to exist, and the decreasing trend was seen in the using of analgesics.

Traditional Chinese herbal medicine has been practiced in China for thousands of years and vast experience has been accumulated for using medicinal herbs for clinical treatment of diseases. Although the biological mechanism of CXDTHFG as traditional Chinese herbal medicine in improving the clinical consequence of migraine is not exactly clear, its analgesic effect and reduction of the days of migraine attacks, frequency, and pain intensity play an important role. First, researchers point out herbal medicines have held a place of importance in remedying headaches effectively and safely [17]. Second, according to the theory of TCM, Rhizoma Chuanxiong (Chuanxiong), originates from the plant Ligusticum chuanxiong Hort, which is used in TCM to "remove blood stasis and expel wind evil to relieve pain." And Uncaria rhynchophylla (Gouteng) can clear liver heat and flatten rising liver yang as well as Chrysanthemums (Juhua). Modern chemical studies indicate that they have the effect of adjusting nerves, antioxidant, anti-inflammatory, sedation, and analgesic [24-26]. Mechanism research of Chinese herbs for the treatment and prophylaxis of migraine should be further studied. 
Overall, the study suggested that CXDT-HFG was efficient in reduction of the frequency of migraine attacks and in alleviating pain degree at week 12, there may be follow-up treatment and prophylaxis effect existing.

4.2. Strengths and Limitations of the Study. Migraine is the patient's subjective feeling, so there may be selective bias in grouping as well as measuring bias and hybrid bias in clinical effect assessment. Thus blinded intervention is necessary [27]. This study is a randomized, double-blind, multicenter, placebo-controlled trial, which can reduce bias factors and improve the reliability and scientific validity of the clinical research.

Migraine has the clinical characteristic of relapsingremitting, and the study mainly chose the remission period for clinical research. Due to the long observation schedule and strict standard of cases inclusion/exclusion, the patients' compliance is poor and future research will be needed to strengthen the management and education of patients to improve the clinical research compliance, and control the rate of dropout.

4.3. Implications for Clinical Practice. Migraine is a recurrent disease and its acute episode and remission period have different pathogenesis characteristics and clinical manifestations. The United States migraine evidence-based guidelines [28] point out that the main goal of acute migraine attacks is to relieve the pain quickly and prevent recurrence; the prevention objective of migraine is to reduce the frequency of migraine attacks and relieve pain severity. The advantage of TCM for migraine lies in the preventative treatment during remission period. CXDT-HFG has certain clinical effect in the preventative treatment of migraine sufferers with "the syndrome of Liver Wind and Blood Stasis," with low adverse reaction. So it is one kind of relatively safe TCM in clinical treatment and prophylaxis for migraine.

4.4. Unanswered Questions and Future Research. The application of TCM must base on the theory of syndrome differentiation and treatment. Patients at different states of migraine need different therapeutic methods. In order to make the clinical efficacy of CXDT-HFG used repeatability, we need to expand sample size and do a larger sample, randomized, double-blind, parallel-controlled clinical research, in which we choose an effective drug for migraine which is accepted internationally as the comparison drug to evaluate the clinical effect characteristics of CXDT-HFG for treatment and prevention of migraine further accurately and objectively.

\section{Conclusion}

CXDT-HFG could reduce the days and frequency of migraine attacks and relieve pain intensity, especially in the prevention of migraine. In addition further research could be conducted on the mechanism of CXDT-HFG for migraine prophylaxis.

\section{Authors' Contribution}

C. Fu and L. Yu contributed equally to this work.

\section{Conflict of Interests}

The authors declare that they have no conflict of interests.

\section{Acknowledgments}

This study was supported by the "Eleventh Five-year Plan" of China State Science and Technology Ministry (2007BAI20B053). The authors are grateful to Professor Liyun He and Statistician Shiyan Yan of the China Academy of Chinese Medical Sciences in Beijing, for helpful comments on the study design and statistical analysis. The authors also appreciate the approval of Hua Run San-Jiu pharmaceutical Co. Ltd for medication supply.

\section{References}

[1] K. Voigt, J. Liebnitzky, U. Burmeister et al., "Efficacy of osteopathic manipulative treatment of female patients with migraine: results of a randomized controlled trial," Journal of Alternative and Complementary Medicine, vol. 17, no. 3, pp. 225-230, 2011.

[2] J. J. Gagne, B. Leas, J. H. Lofland, N. Goldfarb, F. Freitag, and S. Silberstein, "Quality of care measures for migraine: a comprehensive review," Disease Management, vol. 10, no. 3, pp. 138-146, 2007.

[3] M. Diamond, C. Dahlöf, G. Papadopoulos, W. Neto, and S. C. $\mathrm{Wu}$, "Topiramate improves health-related quality of life when used to prevent migraine," Headache, vol. 45, no. 8, pp. 10231030, 2005.

[4] P. J. Goadsby, R. B. Lipton, and M. D. Ferrari, "Migrainecurrent understanding and treatment," New England Journal of Medicine, vol. 346, no. 4, pp. 257-270, 2002.

[5] B. Engmann, "Bipolar affective disorder and migraine," Case Reports in Medicine, vol. 2012, Article ID 389851, 3 pages, 2012.

[6] M. E. Bigal and R. B. Lipton, "The epidemiology, burden, and comorbidities of migraine," Neurologic Clinics, vol. 27, no. 2, pp. 321-334, 2009.

[7] M. Menken, T. L. Munsat, and J. F. Toole, "The global burden of disease study: Implications for neurology," Archives of Neurology, vol. 57, no. 3, pp. 418-420, 2000.

[8] Headache Classification Subcommittee of the International Headache Society, "The international classification of headache disorders: 2nd edition," Cephalalgia, vol. 24, supplement 1, pp. 9-160, 2004.

[9] D. L. Campinha-Bacote, J. B. Kendle, C. Jones et al., "Impact of a migraine management program on improving health outcomes," Disease Management, vol. 8, no. 6, pp. 382-391, 2005.

[10] P. J. Goadsby and T. Sprenger, "Current practice and future directions in the prevention and acute management of migraine," The Lancet Neurology, vol. 9, no. 3, pp. 285-298, 2010.

[11] N. M. Ramadan, S. D. Silberstein, F. G. Freitag et al., "Evidence based guidelines for migraine headache in the primary 
care setting: pharmacological management for prevention of migraine," 2007, http://www.aan.com/professionals/practice/ pdfs/gl0090.pdf.

[12] S. D. Silberstein, "Preventive treatment of migraine," Trends in Pharmacological Sciences, vol. 27, no. 8, pp. 410-415, 2006.

[13] R. B. Lipton, W. F. Stewart, R. Cady et al., "Sumatriptan for the range of headaches in migraine sufferers: results of the spectrum study," Headache, vol. 40, no. 10, pp. 783-791, 2000.

[14] P. Irimia, J. A. Palma, R. Fernandez-Torron, and E. MartinezVila, "Refractory migraine in a headache clinic population," BMC Neurology, vol. 11, article 94, 2011.

[15] A. I. Scher, W. F. Stewart, and R. B. Lipton, "Caffeine as a risk factor for chronic daily headache: a population-based study," Neurology, vol. 63, no. 11, pp. 2022-2027, 2004.

[16] P. J. Goadsby, "Is medication-overuse headache a distinct biological entity?” Nature Clinical Practice Neurology, vol. 2, no. 8 , p. 401, 2006.

[17] H. S. Boon, D. C. Cherkin, J. Erro et al., "Practice patterns of naturopathic physicians: results from a random survey of licensed practitioners in two US States," BMC Complementary and Alternative Medicine, vol. 4, article 14, 2004.

[18] L. P. Wang, X. Z. Zhang, J. Guo et al., "Efficacy of acupuncture for migraine prophylaxis: a single-blinded, double-dummy, randomized controlled trial," Pain, vol. 152, no. 8, pp. 1864 1871,2011

[19] L. P. Wang, X. Z. Zhang, J. Guo et al., "Efficacy of acupuncture for acute migraine attack: a multicenter single blinded, randomized controlled Trial," Pain Medicine, vol. 13, no. 5, pp. 623-630, 2012.

[20] P. Tfelt-Hansen, G. Block, C. Dahlöf et al., "Guidelines for controlled trials of drugs in migraine: second edition," Cephalalgia, vol. 20, no. 9, pp. 765-786, 2000.

[21] World Medical Association General Assembly, "World Medical Association Declaration of Helsinki: ethical principles for medical research involving human subjects," Journal International de Bioéthique, vol. 15, no. 1, pp. 124-129, 2004.

[22] Y. Nestoriuc, A. Martin, W. Rief, and F. Andrasik, "Biofeedback treatment for headache disorders: a comprehensive efficacy review," Applied Psychophysiology Biofeedback, vol. 33, no. 3, pp. 125-140, 2008.

[23] C. T. Hartrick, J. P. Kovan, and S. Shapiro, "The numeric rating scale for clinical pain measurement: a ratio measure," Pain Practice, vol. 3, no. 4, pp. 310-316, 2003.

[24] L. Xiong, Z. Y. Fang, X. N. Tao, M. Bai, and G. Feng, "Effect and mechanism of ligustrazine on Th1/Th2 cytokines in a rat asthma model," American Journal of Chinese Medicine, vol. 35, no. 6, pp. 1011-1020, 2007.

[25] C. L. Chang, C. S. Lin, and G. H. Lai, "Phytochemical characteristics, free radical scavenging activities, and neuroprotection of five medicinal plant extracts," Evidence-Based Complementary and Alternative Medicine, vol. 2012, Article ID 984295, 8 pages, 2012.

[26] J. W. Kim, J. Y. Han, J. T. Hong et al., "Ethanol extract of the flower Chrysanthemum morifolium augments pentobarbital-induced sleep behaviors: involvement of $\mathrm{Cl}^{-}$ channel activation," Evidence-Based Complementary and Alternative Medicine, vol. 2011, Article ID 109164, 7 pages, 2011.

[27] S. J. Day and D. G. Altman, "Blinding in clinical trials and other studies," British Medical Journal, vol. 321, no. 7259, p. $504,2000$.
[28] S. D. Silberstein and W. Edlund, "Practice parameter: evidence-based guidelines for migraine headache (an evidencebased review): report of the Quality Standards Subcommittee of the American Academy of Neurology," Neurology, vol. 55, no. 6, pp. 754-762, 2000. 


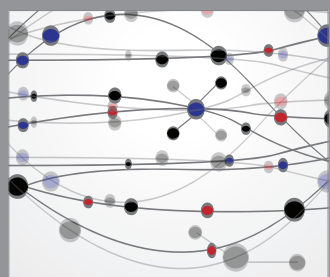

The Scientific World Journal
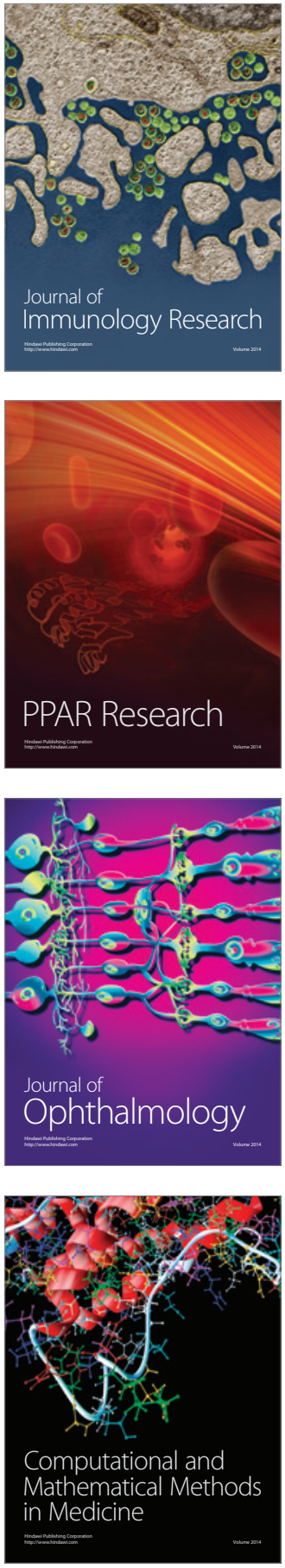

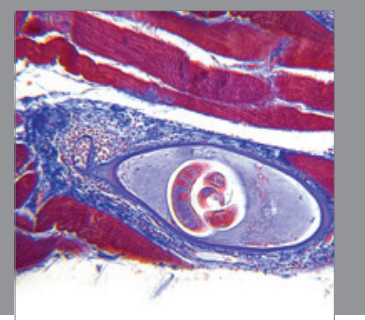

Gastroenterology

Research and Practice
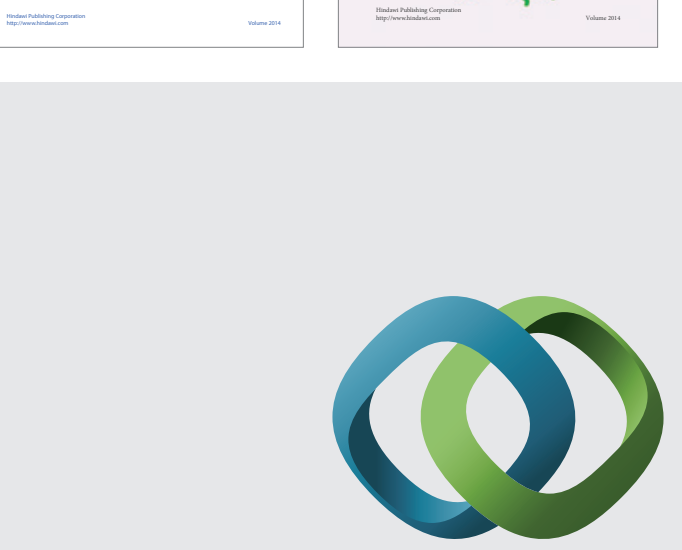

\section{Hindawi}

Submit your manuscripts at

http://www.hindawi.com
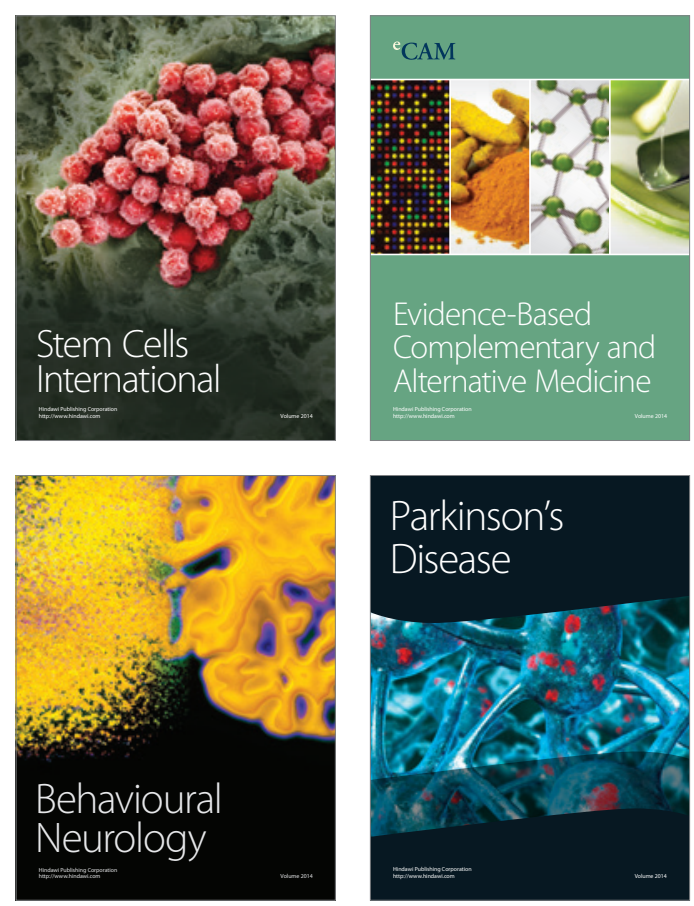

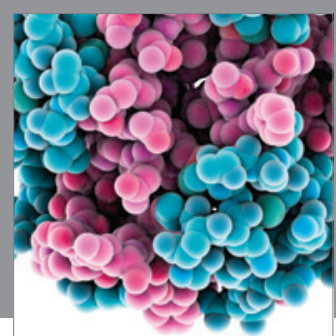

Journal of
Diabetes Research

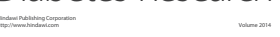

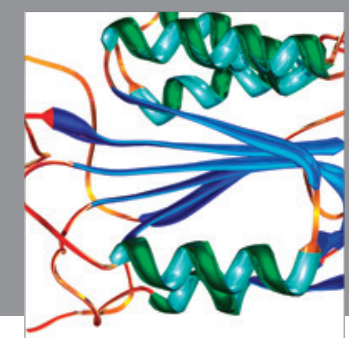

Disease Markers
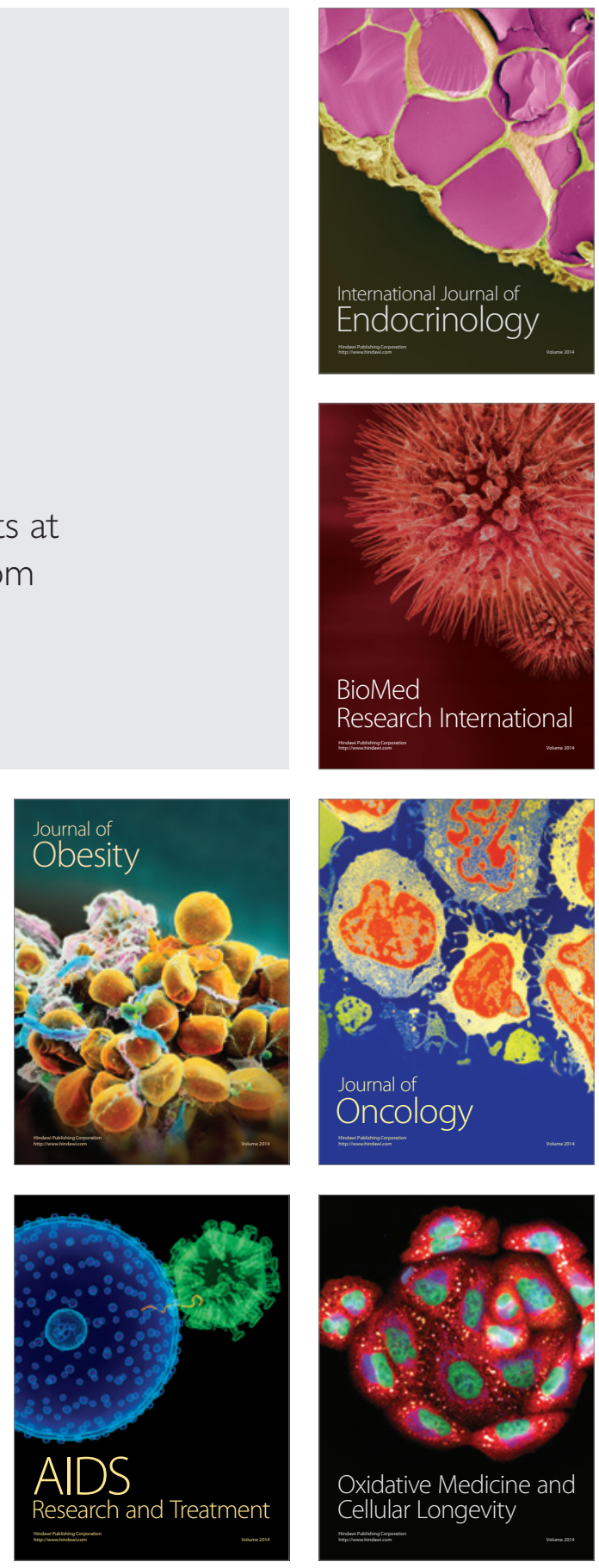